\title{
Exploring Reionization-era Quasars. IV. Discovery of Six New $z \gtrsim 6.5$ Quasars with DES, VHS, and unWISE Photometry
}

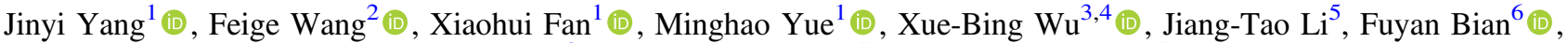 \\ Linhua Jiang $^{3}$ (i), Eduardo Bañados ${ }^{7}$ (10), and Yuri Beletsky ${ }^{7}$ \\ ${ }^{1}$ Steward Observatory, University of Arizona, 933 N. Cherry Avenue, Tucson, AZ, USA; jinyiyang@email.arizona.edu \\ ${ }^{2}$ Department of Physics, University of California, Santa Barbara, CA 93106-9530, USA \\ ${ }^{3}$ Kavli Institute for Astronomy and Astrophysics, Peking University, Beijing 100871, People's Republic of China \\ ${ }^{4}$ Department of Astronomy, School of Physics, Peking University, Beijing 100871, People's Republic of China \\ ${ }^{5}$ Department of Astronomy, University of Michigan, 311 West Hall, 1085 S. University Avenue, Ann Arbor, MI 48109-1107, USA \\ ${ }^{6}$ European Southern Observatory, Alonso de Córdova 3107, Casilla 19001, Vitacura, Santiago 19, Chile \\ ${ }^{7}$ The Observatories of the Carnegie Institution for Science, 813 Santa Barbara Street, Pasadena, CA 91101, USA \\ Received 2018 November 28; revised 2019 April 22; accepted 2019 April 23; published 2019 May 21
}

\begin{abstract}
This is the fourth paper in a series of publications aiming at discovering quasars at the epoch of reionization. In this paper, we expand our search for $z \sim 7$ quasars to the footprint of the Dark Energy Survey (DES) Data Release One (DR1), covering $\sim 5000 \mathrm{deg}^{2}$ of a new area. We select $z \sim 7$ quasar candidates using deep optical, near-infrared (near-IR) and mid-infrared (mid-IR) photometric data from the DES DR1, the VISTA Hemisphere Survey, the VISTA Kilo-degree Infrared Galaxy survey, the UKIRT InfraRed Deep Sky Surveys-Large Area Survey (ULAS), and the unblurred coadds from the Wide-field Infrared Survey Explore (WISE) images (unWISE). The inclusion of DES and unWISE photometry allows the search to reach $\sim 1$ mag fainter, comparing to our $z \gtrsim 6.5$ quasar survey in the northern sky. We report the initial discovery and spectroscopic confirmation of six new luminous quasars at $z>6.4$, including an object at $z=7.02$, the fourth quasar yet known at $z>7$, from a small fraction of candidates observed thus far. Based on the recent measurement of $z \sim 6.7$ quasar luminosity function using the quasar sample from our survey in the northern sky, we estimate that there will be $\gtrsim 55$ quasars at $z>6.5$ at $M_{1450}<-24.5$ in the full DES footprint.
\end{abstract}

Key words: galaxies: active - galaxies: high-redshift - quasars: emission lines

\section{Introduction}

Luminous quasars at high redshift provide direct probes of the evolution of supermassive black holes (BHs) and the intergalactic medium (IGM) at early cosmic time. Over the last decade, more than 150 quasars have been discovered at $z>6$ (e.g., Fan et al. 2001, 2006; Willott et al. 2010; Mortlock et al. 2011; Venemans et al. 2013, 2015; Kashikawa et al. 2015; Reed et al. 2015, 2017; Wu et al. 2015; Bañados et al. 2016, 2018; Jiang et al. 2016; Matsuoka et al. 2016; Mazzucchelli et al. 2017; Wang et al. 2017; Matsuoka et al. 2018a, 2018b), with the highest redshift at $z=7.54$ (Bañados et al. 2018). Detections of such objects indicate the existence of billion $M_{\odot}$ BHs merely a few hundred Myr after the first star formation in the universe. It challenges the theory of $\mathrm{BH}$ growth and places the strongest constraints on the BH-galaxy coevolution at early epoch (e.g., Wu et al. 2015). Absorption spectra of the highest redshift quasars reveal complete GunnPeterson absorption, indicating a rapid increase in the IGM neutral fraction, marking the end of the reionization epoch at $z>6$ (see Fan et al. 2006). Combined with results from the most recent detections on the declining $\operatorname{Ly} \alpha$ visibility and abundance among high-redshift galaxies and revised measurements of Thompson optical depth from cosmic microwave background $(\mathrm{CMB})$ polarization, current data strongly suggest a peak of reionization activity and emergence of the earliest galaxies and active galactic nuclei (AGNs) at $6<z<10$ (Robertson et al. 2015; Planck Collaboration et al. 2018), highlighting the need to expand our search to higher redshift.

At $z \gtrsim 7$, the $\operatorname{Ly} \alpha$ emission line is redshifted to beyond 1 micron, requiring near-infrared (near-IR) observations for both their selections and spectroscopic identifications. Efforts to find such objects have proven to be difficult. Until recently, only two quasars have been discovered at $z \gtrsim 7$, and a dozen at $z>6.5$, compared to the large number of available objects at $z \sim 6$, despite a decade-long effort by the high-redshift quasar community. This is due to a combination of their declining spatial density, limited sky coverage of near-IR photometry, and low efficiency in spectroscopic follow-up observations. This situation is rapidly changing, with new deep optical and infrared (IR) sky survey data finally becoming available. In the optical, the Dark Energy Spectroscopic Instrument Legacy Imaging Surveys (DELS; Dey et al. 2019) is covering the Sloan Digital Sky Survey (SDSS) footprint with high-quality, deep photometry in the $g, r$, and $z$ bands, the Pan-STARRS1 (PS1; Chambers et al. 2016) survey provides $g, r, i, z$, and $y$ observations over a $3 \pi$ area of the sky, and the Dark Energy Survey (DES; Abbott et al. 2018) DR1 reaches even fainter flux levels in the $g, r, i, z$, and $Y$ bands over $5000 \mathrm{deg}^{2}$ in the southern sky; in the near-IR, the United Kingdom Infrared Telescope (UKIRT) Hemisphere Survey (UHS; Dye et al. 2018), the UKIRT InfraRed Deep Sky Surveys-Large Area Survey (ULAS; Lawrence et al. 2007), the VISTA Hemisphere Survey (VHS; McMahon et al. 2013), and the VISTA Kilodegree Infrared Galaxy survey (VIKING; Edge et al. 2013) are mapping almost the entire sky; and in the mid-IR, the latest Wide-field Infrared Survey Explore (WISE; Wright et al. 2010) survey from the Near-Earth Object WISE (NEOWISE; Mainzer et al. 2011, 2014) project has doubled the depth in the critical $W 1$ and $W 2$ bands. The unblurred coadds of WISE (unWISE; Lang 2014) have better resolution and enhanced depths than 
Table 1

Photometric Data Used in This Survey

\begin{tabular}{lccc}
\hline \hline Survey & Area $\left(\mathrm{deg}^{2}\right)$ & Data Used & Depth $(5 \sigma)$ \\
\hline DES DR1 & 5000 & $g, r, i, z, Y$ & $25.08,24.83,24.19$, \\
& & & $23.44,22.19$ \\
VHS DR5 $^{\mathrm{a}}$ & 3600 & $J, K s$ & $20.2,19.2,18.2$ (Vega) \\
VIKING DR4 $^{\text {ULAS DR10 }}$ & 300 & $J, K s$ & $21.2,19.4$ (Vega) \\
unWISE & 170 & $J, K$ & $19.6,18.2$ (Vega) \\
& all-sky & $W 1, W 2$ & $18.3^{\mathrm{b}}, 16.9$ (Vega) \\
\hline
\end{tabular}

Notes.

${ }^{\text {a }}$ We only show the near-IR-DES overlapped area here.

b The unWISE depth is estimated from the magnitude-error relation of unWISE data in the DES area.

blurred ALLWISE data in the it $W 1 / W 2$ bands with new releases of the additional data from the NEOWISE-Reactivation (NEOWISER; Mainzer et al. 2014) phase of the mission (Meisner et al. 2017, 2018).

The combination of these surveys allows the optical/near-IR selection of quasars at $z \gtrsim 6.5$ in deeper and wider field sky than in the past. Our group is conducting a survey of quasars at $z \gtrsim 6.5$ (Wang et al. 2017, 2018a, 2018b; Fan et al. 2019) utilizing these new data sets. Wang et al. (2018b, hereafter Paper III) present the discovery of 16 new quasars from our survey in the northern sky using the combination of DELS, PS1, UHS, ULAS, VHS, VIKING, and WISE data; we have doubled the number of known quasars at $z>6.5$, measured the quasar luminosity function (QLF) at $z=6.7$, and constrained the quasar number density evolution during the reionzation epoch. In this paper, we present our ongoing survey in the southern sky based on a similar optical/IR selection method, using the data from DES, VHS, VIKING, and unWISE, and report the initial discoveries of six new quasars at $6.41 \leqslant z \leqslant 7.02$ based on a small fraction of candidates observed. We introduce the photometric data set and quasar selection criteria used in this survey in Section 2. The spectroscopic observations and the result are described in Sections 3 and 4. We discuss the implication of these discoveries and conclude with a short summary in Section 5. In this paper, we adopt a $\Lambda$ CDM cosmology with parameters $\Omega_{\Lambda}=0.7, \Omega_{m}=0.3$, and $H_{0}=70 \mathrm{~km} \mathrm{~s}^{-1} \mathrm{Mpc}^{-1}$. Photometric data from DES are reported on the $\mathrm{AB}$ system after applying the Galactic extinction correction (Schlegel et al. 1998; Schlafly \& Finkbeiner 2011; Abbott et al. 2018); photometric data from IR (e.g., VHS, VIKING, ULAS, and WISE) surveys are reported in the Vega system.

\section{Candidate Selection}

\subsection{Photometric Data Sets}

This survey is a part of our wide-field $z \gtrsim 6.5$ quasar survey based on optical/IR photometry. Similar to our survey in the northern sky in Paper III, we are mainly using colors in the $z, Y$, $J$, and $W 1$ bands but modify the selection criteria according to the DES filters. The photometric data is from DES DR1, VHS, VIKING, ULAS, and unWISE. Except for the $\sim 170 \mathrm{deg}^{2}$ field that is only covered by ULAS without VHS images, photometric data used here is deeper than the optical/IR data used for our survey in the northern sky, which enables us to search quasars with $\gtrsim 1$ mag fainter. A summary of photometric data used in this survey is shown in Table 1.
In the optical, we use DES DR1 photometric data. The DES survey uses the Dark Energy Camera (DECam; Honscheid et al. 2008; Flaugher et al. 2015) on the CTIO $4 \mathrm{~m}$ Blanco Telescope to image five broad bands $(g, r, i, z$, and $Y)$ in a 5000 $\mathrm{deg}^{2}$ field in the southern Galactic cap. The DR1 data encompasses images from first three years of the survey, with a coadd magnitude limit (MAG_APER_4, 1.95 arcsec diameter, $10 \sigma$ ) of $24.33,24.08,23.44,22.69$, and 21.44 in the five bands, respectively. In particular, the DES $Y$ band covers the wavelength from 9300 to $10700 \AA$ extended to the near-IR wavelength range. It reaches $\sim 1$ mag fainter than $z$ in the PS1 survey, 0.6 mag fainter than $z$ in DELS (DR4+DR5), and covers a redder wavelength range $(\sim 700 \AA)$ than PS1 $z$. DES $z$ imaging allows the detection of quasar at redshift up to $z \sim 7.2$.

In the near-IR, we are using all public near-IR images in the DES area, including those from the VHS, VIKING, and ULAS. The VHS survey aims at imaging the entire hemisphere of the southern sky in the $Y, J, H$, and $K s$ bands, with the depth $(5 \sigma)$ to 20.6, 20.2, 19.2, and $18.2 \mathrm{mag}$ in Vega. The VHS-DES area will be $\sim 4400 \mathrm{deg}^{2}$, while the current VHS DR5 has covered $\sim 80 \%$ of the entire VHS-DES area. The VIKING survey covers $\sim 1500 \mathrm{deg}^{2}$ in the $Z, Y, J, H$, and $K s$ bands over three extragalactic areas, with the $5 \sigma$ depth of $22.6,21.7,21.2,20.1$, and $19.4 \mathrm{mag}$. The entire VIKING-DES area is about 500 $\mathrm{deg}^{2}$, of which about $300 \mathrm{deg}^{2}$ is available now. The ULAS survey has a $\sim 450 \mathrm{deg}^{2}$ overlap region with the DES, among which a $\sim 280 \mathrm{deg}^{2}$ field is also covered by the VHS. For the area covered by both VHS and ULAS we use the VHS data as it is deeper than ULAS. The survey depth of ULAS in the $Y, J$, $H$, and $K$ bands is 20.2, 19.6, 18.8, and $18.2 \mathrm{mag}$.

Discoveries presented in this paper used the DES $Y$ band as the detection band. To improve the signal-to-noise ratio $(\mathrm{S} / \mathrm{N})$ of faint objects, we carried out forced photometry of each object in the $J$ and $K s(K)$ band centered on the position from DES DR1. We used a 2-arcsec aperture diameter, the same to that used for JAperMag3 in the VHS, VIKING, and ULAS surveys. The forced photometry also provides measurements for faint objects that are covered by near-IR imaging but are below the detection limit of the public catalog. The $J$ and $K s$ $(K)$ band magnitudes used in the color selection in Section 2.2 are our forced photometric magnitudes for all objects.

Photometric data in the WISE $W 1$ and $W 2$ bands is useful to separate quasars from brown dwarfs (e.g., the $W 1-W 2$ color). Compared with the deep DES, VHS, and VIKING surveys, the ALLWISE data with the depth $(5 \sigma)$ of 17.6 and 16.2 mag is relatively shallow, with position-dependent depth (coverage). Therefore, we performed forced photometry on the unWISE coadded image of each object using the DES position. We used the forced photometry code from the Tractor (Lang et al. 2016a, 2016b). The current unWISE coadded image incorporates WISE and three years of NEOWISER imaging. The forced photometric unWISE data in the DES area is $\sim 0.7 \mathrm{mag}$ deeper than ALLWISE, based on the magnitude-error relation of the forced photometric data in the DES area.

\subsection{Selection Criteria}

We started our selection with the DES DR1 catalog (Abbott et al. 2018) and used the MAG_APER_4 mag. We first selected objects that had $>5 \sigma$ detection in the $Y$ band but were undetected in the $g$ and $r$ bands with $\mathrm{S} / \mathrm{N}<3$. We require the objects to be undetected in the $i$ band $(\mathrm{S} / \mathrm{N}(i)<3)$ or with $i-z>3$ color. The former cut is used to select the fully $i$ 

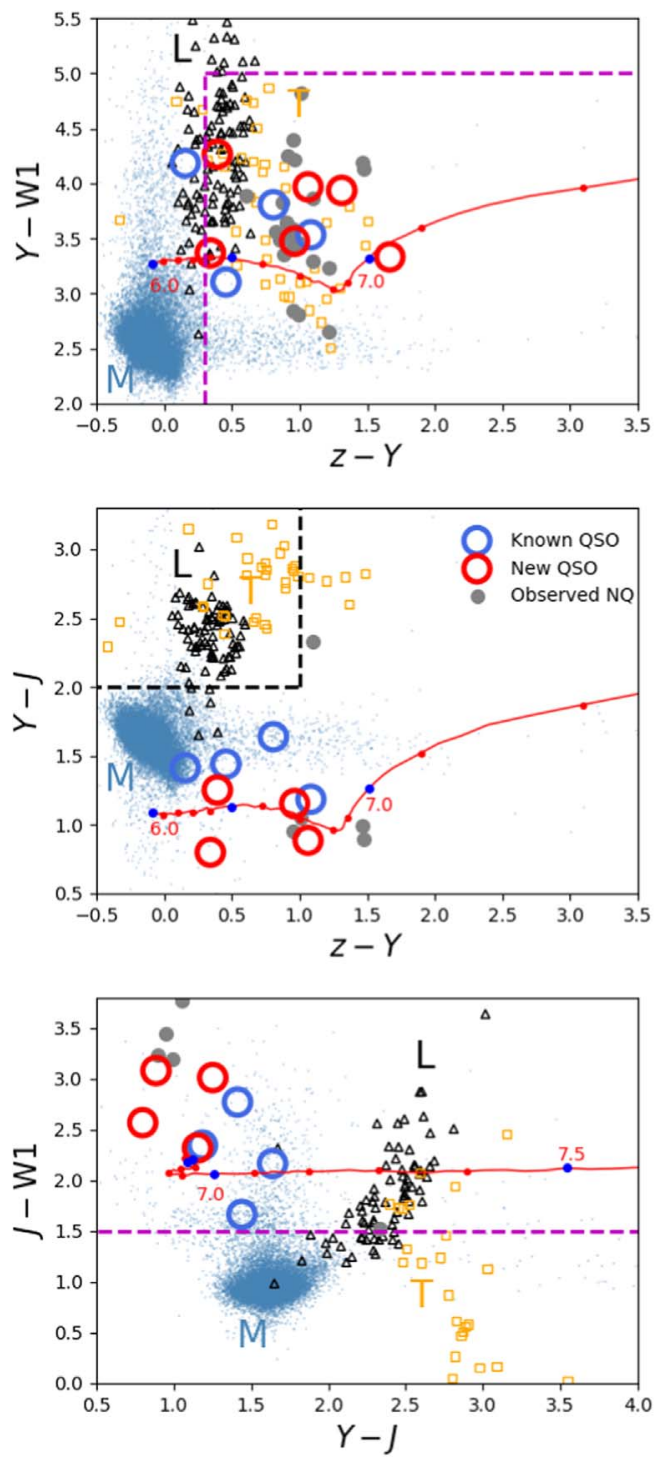

Figure 1. Quasar selection color-color cuts, in $z-Y / Y-W 1$, (top) $z-Y / Y-J$ (middle), and $Y-J / J-W 1$ (bottom) color-color diagrams. The red solid lines with the red/blue points are the quasar color track started at $z=6.0$ with the step of $\Delta z=0.1$. The blue points mark the redshifts of $z=6.0,6.5,7.0$, and 7.5. The blue open circles represent colors of four previously known $z \geqslant 6.5$ quasars in the DES DR1. The red open circles denote the six new quasars presented in this paper. For $J$ and $W 1$, forced photometry measurements are used. Quasars J025216-050331 and J031941-100846 do not have near-IR images and thus there are only four red open circles in the $z-Y / Y-J$ and $Y-J / J-W 1$ diagrams. The purple/black dashed lines represent the selection/rejection criteria. The gray filled circles are observed candidates but identified as non-quasar (NQ) objects (see Section 4).

band drop-out objects (we call it the main sample), and the latter one is designed for ultra-luminous quasars that are still detected in the deep DES $i$ band (luminous sample). We used the baseline quality criterion of IMAFLAGS_ISO $=0$ (Abbott et al. 2018) for DES photometry. We selected objects in the $z-Y / Y-W 1$ color space for quasars at $z \gtrsim 6.5$ and reject most of the $\mathrm{M}$ dwarfs and part of the L, T dwarfs, as shown in Figure 1 . We also limit objects with $0<W 1-W 2<2$. The typical $W 1-W 2$ cut used for quasar selection is $W 1-W 2>0.5$, except for the quasar at $z \sim 4.4-4.7$ where the $W 1-W 2$ drops to $>0.2$ (Wang et al. 2016). The unWISE $W 2$ has relatively large uncertainty. Thus we loosened the
$W 1-W 2$ cut to $W 1-W 2>0$. The upper limit of $W 1-W 2<2$ is used to reject extremely red $\mathrm{L} / \mathrm{T}$ dwarfs. Then we cross-matched ( $2^{\prime \prime}$ radius) the color-selected candidates with PS1 DR1 and did forced photometry in the PS1 $g, r$, $i$, and $z$ bands using a $2^{\prime \prime}$ diameter aperture to further reduce candidates. We reject objects with $i_{\mathrm{ps} 1 \text {,forced }}$ brighter than 23.1 mag or $z_{\text {ps1,forced }}$ brighter than 22.3 mag in the main sample, and only limit the $i_{\mathrm{ps} 1 \text {, forced }}$ for objects in the luminous sample. The selection criteria are listed as follows. DES $g, r, i, z$, and $Y$ magnitudes are in the $\mathrm{AB}$ system, while the IR $J, K, W 1$, and $W 2$ are in Vega system with the Vega to AB conversions of 0.937 (0.938 for ULAS $J), 1.839$ (1.900 for ULAS $K$ ), 2.699, and 3.339 , respectively.

$$
\begin{aligned}
& \mathrm{S} / \mathrm{N}(g)<3.0, \mathrm{~S} / \mathrm{N}(r)<3.0, \mathrm{~S} / \mathrm{N}(Y)>5.0 \\
& \text { Main: } \mathrm{S} / \mathrm{N}(i)<3.0 \\
& \text { Luminous: } \mathrm{S} / \mathrm{N}(i) \geqslant 3.0, i-z>3.0 .
\end{aligned}
$$

After the baseline quality criterion and the $\mathrm{S} / \mathrm{N}$ cuts, we obtain $\sim 19,000$ and 160 candidates in the main and luminous samples, respectively.

$$
\begin{gathered}
z-Y>0.3 \text { and } Y-W 1<5 \\
0<W 1-W 2<2 \\
\text { Main: } i_{\mathrm{ps} 1, \text { forced }}>23.1 \text { and } z_{\mathrm{ps} 1 \text {,forced }}>22.3
\end{gathered}
$$$$
\text { Luminous: } i_{\mathrm{ps} 1 \text {,forced }}>23.1 \text {. }
$$

After these selections, there are $\sim 3500$ candidates in the main sample and $\sim 80$ candidates in the luminous sample. To further reject contaminants, we add near-IR $J$ and $K s(K)$ band data from VHS, VIKING, and ULAS based on forced photometry measurements described above. Since the near-IR surveys do not fully cover the DES area, we kept all objects that do not have $J$ or $K s$ images. We used the $z-Y / Y-J$ and $J-W 1$ colors for objects rejection, as shown in Figure 1. We rejected all objects which had a $J$ band image but with negative flux from the forced photometry. A $J-K s / K$ cut was added for objects that had $K s(K)$ detection, which is designed for further rejection based on the power-law continuum of a quasar. We rejected objects that satisfied any one of the criterion listed below.

$$
\begin{gathered}
z-Y<1 \text { and } Y-J>2 \\
J-W 1<1.5 \\
J-K s<0.5
\end{gathered}
$$

After applying all of the selection criteria above, we obtained $\sim 2100$ candidates in the main sample and $\sim 40$ candidates in the luminous sample. We then visually inspected the optical/IR image of each candidate. We rejected extended objects, objects visible in any of the DES $g, r, i$ (except those with $i$ band detection in the luminous sample) and PS1 $g, r, i$ bands, and objects with photometry contaminated by cosmic rays or nearby bright stars. We finally obtained a candidate sample including $\sim 380$ candidates, 350 from the main sample and 30 from the luminous sample. In the DES area, there are five previously known quasars at $z \geqslant 6.5$ (Venemans et al. 2013, 2015; Reed et al. 2017; Matsuoka et al. 2018a). Four quasars are included in the DES DR 1 catalog, except for the faint quasar HSC J0213-0626 (Matsuoka et al. 2018a). Among these four known quasars, three are covered by our selection: $\mathrm{J} 010953-304736$ at $z=6.79, \mathrm{~J} 030516-315056$ at $z=6.61$ (Venemans et al. 2013), and PSO J036+03 (also named as J022601.873+030259.254; Venemans et al. 2015). The quasar 
Table 2

Photometric Data of Six New Quasars

\begin{tabular}{|c|c|c|c|c|c|c|c|c|c|}
\hline Name & Redshift & $M_{1450}$ & $\bar{z}$ & 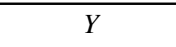 & $\overline{J^{\mathrm{a}}}$ & 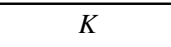 & "NIR & $W 1^{\mathrm{b}}$ & W2 \\
\hline DES J021638.85-522620.6 & 6.41 & $-25.19 \pm 0.19$ & $22.11 \pm 0.06$ & $21.72 \pm 0.13$ & $20.47 \pm 0.19$ & $18.95 \pm 0.24$ & VHS & $17.46 \pm 0.05$ & $17.13 \pm 0.15$ \\
\hline DES J024655.90-521949.9 & 6.87 & $-25.35 \pm 0.21$ & $22.30 \pm 0.15$ & $21.24 \pm 0.14$ & $20.35 \pm 0.20$ & $18.26 \pm 0.18$ & VHS & $17.27 \pm 0.04$ & $16.76 \pm 0.11$ \\
\hline DES J025216.64-050331. $8^{\mathrm{c}}$ & 7.02 & $-26.50 \pm 0.09$ & $22.52 \pm 0.10$ & $20.85 \pm 0.07$ & $19.25 \pm 0.07$ & $\ldots$ & UKIRT & $17.52 \pm 0.08$ & $16.98 \pm 0.22$ \\
\hline DES J031941.66-100846.0 & 6.83 & $-25.71 \pm 0.20$ & $22.35 \pm 0.13$ & $21.04 \pm 0.20$ & $\ldots$ & $\ldots$ & $\ldots$ & $17.11 \pm 0.04$ & $16.12 \pm 0.08$ \\
\hline DES J213110.29-435902.5 & 6.45 & $-24.93 \pm 0.25$ & $21.89 \pm 0.05$ & $21.54 \pm 0.10$ & $20.74 \pm 0.25$ & $18.91 \pm 0.24$ & VHS & $18.17 \pm 0.13$ & $17.49 \pm 0.30$ \\
\hline DES J221100.60-632055.8 & 6.88 & $-25.10 \pm 0.23$ & $22.72 \pm 0.10$ & $21.76 \pm 0.13$ & $20.60 \pm 0.22$ & $19.22 \pm 0.35$ & VHS & $18.28 \pm 0.14$ & $18.02 \pm 0.44$ \\
\hline
\end{tabular}

Notes.

${ }^{\text {a }}$ Forced photometric data of the $J$ and $K s$ band images from a public survey.

b Forced photometry on unWISE coadds.

${ }^{\mathrm{c}}$ Quasar J025216-050331 and J031941.66-100846.0 do not have public near-IR imaging data. The $J$ band data of J025216-050331 is from our recent UKIRT observation after the discovery. 
Table 3

Spectroscopy Information of the Six New Quasars

\begin{tabular}{|c|c|c|c|c|c|c|c|}
\hline Name & Redshift & $M_{1450}$ & Instrument & Exp. Time & Grating & Slit & $\overline{\text { Obs. Date }}$ \\
\hline J021638.85-522620.6 & 6.41 & $-25.19 \pm 0.19$ & Magellan/LDSS3 & 1800 & VPH-Red & 1.25_Center & $2018 \mathrm{Jul} 21$ \\
\hline J024655.90-521949.9 & 6.87 & $-25.35 \pm 0.21$ & Magellan/LDSS3 & 1500 & VPH-Red & 1.25_Center & 2018 Jul 22 \\
\hline \multirow[t]{2}{*}{ J025216.64-050331.8 } & 7.02 & $-26.50 \pm 0.09$ & Magellan/LDSS3 & 1200 & VPH-Red & 1.25_Center & 2018 Jul 22 \\
\hline & & & Gemini/GMOS-S & 8000 & $\mathrm{R} 400$ & 0".75longslit & 2018 Oct 1 \\
\hline J031941.66-100846.0 & 6.83 & $-25.71 \pm 0.20$ & MMT/Binospec & 1800 & 270 lines $/ \mathrm{mm}$ & 1 ".25 long-slit & 2018 Nov 8 \\
\hline $\mathrm{J} 213110.29-435902.5$ & 6.45 & $-24.93 \pm 0.25$ & Magellan/FIRE & 600 & $\ldots$ & 1"0_longslit & 2018 May 2 \\
\hline $\mathrm{J} 221100.60-632055.8$ & 6.88 & $-25.10 \pm 0.23$ & Magellan/LDSS3 & 5400 & VPH-Red & 1.25_Center & 2018 Jul 21 \\
\hline
\end{tabular}

J022426-471129 at $z=6.5$ from Reed et al. (2017) was rejected by the $z-Y>0.3$ cut.

\section{Spectroscopic Observations}

We conducted spectroscopic observations of these DESselected candidates with Magellan/FIRE (Simcoe et al. 2008), Magellan/LDSS3-C (Stevenson et al. 2016), and MMT/ Binospec (Fabricant et al. 1998) in 2018 May, July, and November. FIRE is an IR echelle/long-slit spectrograph on the Magellan Baade $6.5 \mathrm{~m}$ telescope in Chile. We used the 1!"0 long-slit mode with FIRE, which provides a resolution of $R \sim 300-500$ covering the wavelength from 8000 to $22000 \AA$. The throughput at wavelengths shorter than $8500 \AA$ is low. We typically use 5-10 minute exposure times for each target. LDSS3 is a high-efficiency spectrograph and optical imager on the Magellan Clay $6.5 \mathrm{~m}$ telescope. We used the VPH-Red grism covering the wavelength range of 6000-10000 $\mathrm{A}$. We used a 1"'25 slit with $R \sim 1000$. The typical exposure time for identification is 20-30 minutes. MMT Binospec is a wide-field optical spectrograph capable of mutli-object spectroscopy, single-slit, and imaging with dual $8^{\prime} \times 15^{\prime}$ fields of view. We used the 270 lines/millimeter grating centered at $7800 \AA$ with a 1 ." 25 slit, which provides a resolution of $R \sim 1200$ and an effective wavelength coverage from 5130 to $10200 \AA$. We observed 31 candidates from the main sample in total. After discovery, we took the follow-up optical spectroscopy for new quasars with LDSS3 and GMOS-S in the Gemini South $8.1 \mathrm{~m}$ telescope to obtain higher quality optical spectra. Data from FIRE, LDSS3, and Binospec was reduced with standard IRAF routines. The GMOS data was reduced using the Gemini IRAF packages.

\section{Results}

At the time of this publication, we have discovered six new quasars at $6.41<z<7.02$ from the DES-near-IR-unWISE selection. Four of the quasars are at $z>6.8$. Quasar J024655.90-521949.9 is also independently discovered by Reed et al. 2019. The photometric properties and spectroscopic information of these six new quasars are listed in Tables 2 and 3. Among other observed candidates, some are L/T dwarfs and some are non-quasar objects that do not have obvious break and broad emission lines but the $\mathrm{S} / \mathrm{Ns}$ are too low for definitive identifications.

We measure the quasar redshifts by visually matching the observed spectrum to the SDSS quasar template (Vanden Berk et al. 2001) using an eye-recognition assistant for the quasar spectra software (ASERA; Yuan et al. 2013). The matching is based on emission lines Ly $\alpha$ and $\mathrm{N} \mathrm{V}$. The typical uncertainty of the redshift measurement is around 0.03 . For spectra of J021638.85-522620.6 and J213110.29-435902.5, due to the low $\mathrm{S} / \mathrm{N}$, the uncertainty could be $\sim 0.05$. We do not include the systematic offset of the $\operatorname{Ly} \alpha$ emission line (e.g., Shen et al. 2007), which is typically $\sim 500 \mathrm{~km} \mathrm{~s}^{-1}$ and much smaller than the uncertainty of template matching. As a test of our redshift measurement applied for high-redshift quasars, using our method we measure redshifts of 18 quasars at $z>6.3$ that have published Mg II-based redshifts (e.g., Mazzucchelli et al. 2017; Reed et al. 2019). Our redshift measurements based on the Ly $\alpha$ and $\mathrm{NV}$ lines are consistent with the Mg II-based redshifts within $\Delta z<0.03$. The spectra of the new quasars are shown in Figure 2.

As the spectra of these six new quasars do not have sufficient wavelength coverage and $\mathrm{S} / \mathrm{N}$ for a reliable continuum fit, we cannot measure the $M_{1450}$, the absolute continuum magnitude at the rest frame $1450 \mathrm{~A}$, directly from the spectra. Therefore, we estimate the $M_{1450}$ using the composite spectra of luminous low-redshift quasars (Selsing et al. 2016). We scale the composite spectrum with the $J$ band photometry of each quasar and then measure the magnitude at $1450 \AA$ from the scaled composite spectrum. For quasar J031941.66-100846.0, we use the $Y$ band data since it does not have $J$ band photometry. The $M_{1450}$ magnitudes of the new quasars are also listed in Tables 2 and 3.

Figure 3 shows the locations of the six new quasars in the $M_{1450}$-redshift plot of all known $z>6.3$ quasars. Compared to Paper III, the sample presented in this paper extends to a fainter population. Although this survey is still ongoing and only a small number of candidates have been observed, we have already discovered five quasars at $z \gtrsim 6.5$, including four at $z>6.8$, deep into the epoch of reionization.

Quasar J025216.64-050331.8 is the fourth known quasar at a redshift higher than 7. Its GMOS-S spectrum (Figure 2) shows a strong absorption profile close to the Ly $\alpha$ emission line. The near-IR spectrum is required for the further investigation of this absorption feature, e.g., whether it is due to the Ly $\alpha$ damping wing from a significantly neutral IGM, is due to a broad absorption line feature, or the presence of a proximate damped Ly $\alpha$ absorber. We are collecting the highquality optical and near-IR spectra of the four new $z>6.8$ quasars for the further studies of their $\mathrm{BH}$ masses and IGM properties.

\section{Discussion and Summary}

The new discoveries, especially the four quasars at $6.83 \leqslant z \leqslant 7.02$, from our DES-near-IR-unWISE quasar selection suggest that the combination of the deep DES optical data, VHS/VIKING near-IR, and unWISE mid-IR data is highly effective in searching for quasars at $z>6.5$. Among our six new quasars, only three have ALLWISE detections, three have $J$ band public data, and none of them have both $J$ and $W 1$ 


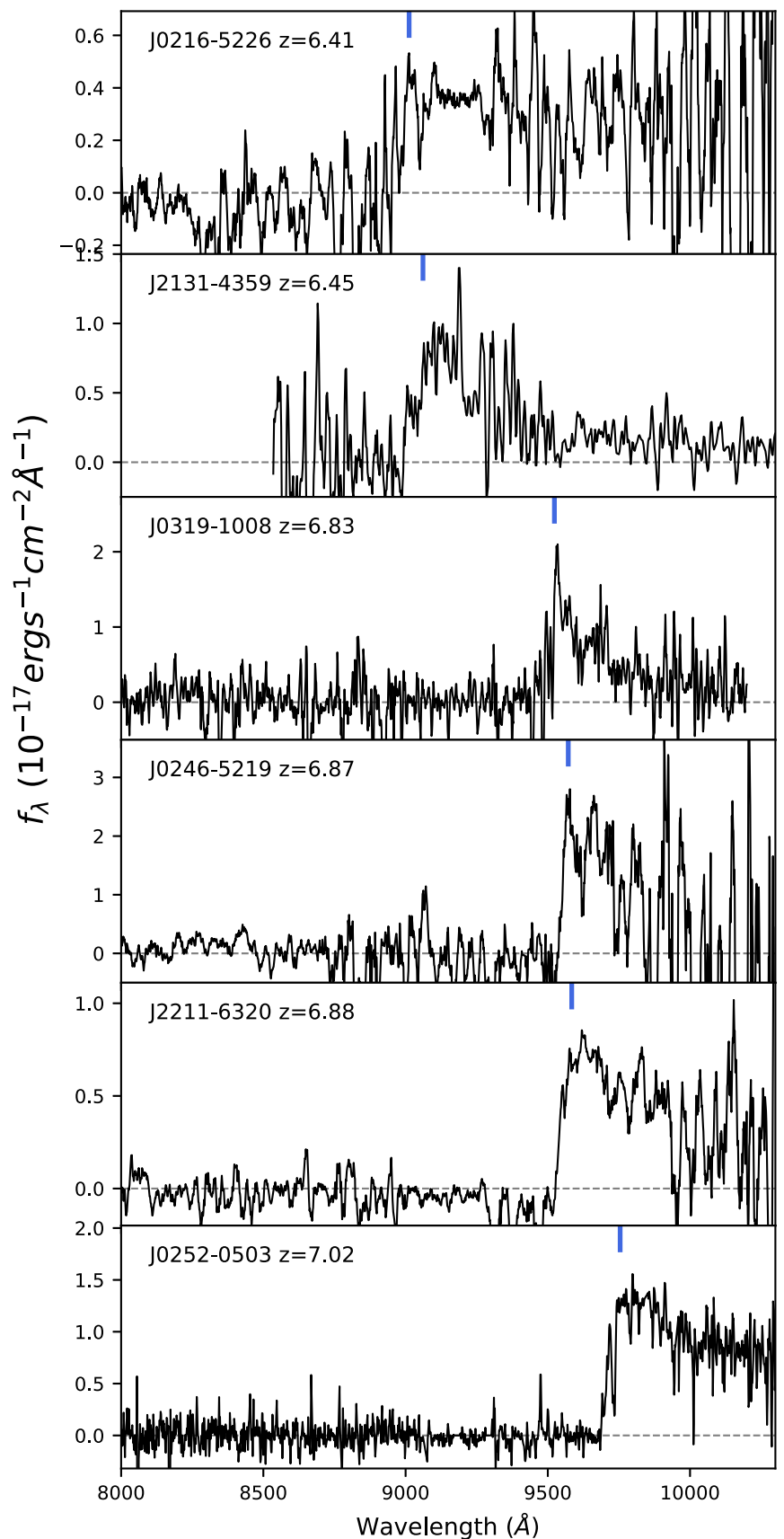

Figure 2. Spectra of the six new $z>6.4$ quasars. The blue vertical lines mark the locations of the Ly $\alpha$ emission line. Spectra from Magellan/LDSS3 are smoothed with a 15 pixel boxcar, the spectrum from Magellan/FIRE is smoothed with a 3 pixel boxcar, and the MMT/Binospec spectrum is smoothed with 5 pixel boxcar. The spectrum of J025216.64-050331.8 is from Gemini GMOS-S. All spectra are corrected for Galactic extinction using the coefficients given by DES DR1 (Abbott et al. 2018) and $E(B-V)$ derived from the Schlegel et al. (1998) dust map.

public data used for the $J-W 1$ cut. The forced photometric data of the near-IR and unWISE images significantly improve the effectiveness of our photometric data set for quasar selection.

With this deep photometric data set, we are able to reach $\sim 1$ mag fainter than our survey in the northern sky. Based on the double power QLF presented in Paper III, we estimate the total number of quasars at $6.5<z<7.1$ and $M_{1450}<-24.5$ in the DES area is around 60. The quasar sample in Paper III

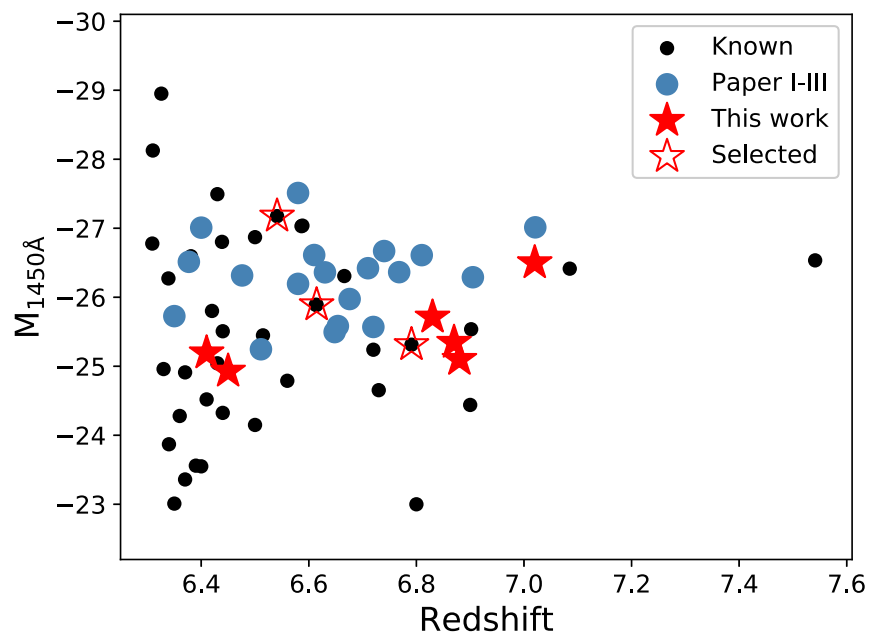

Figure 3. Redshift and $M_{1450}$ distribution of quasars at $z \geqslant 6.3$. The black filled circles are the previously known quasars. The blue filled circles denote quasars from our surveys in the northern sky (Wang et al. 2017, 2018a, 2018b, Papers I, II, and III). The red filled stars represent the six new quasars from this survey and the red open stars are the three known quasars in the DES area that meet our selection criteria.

only covers the relatively bright quasars with $M_{1450}<-25.5$ and the luminosity function is measured with fixed faint end slope $(\alpha=-1.90)$ and break magnitude $\left(M^{*}=-25.2\right)$ from the previous $z \sim 6$ QLF (Jiang et al. 2016). However, the quasar sample from Jiang et al. (2016) only includes two quasars at $M_{1450}>-24$ and thus the faint end slope and break magnitude are less well constrained. As a comparison, we estimate the number based on different QLFs. Using the single power-law fit $(\beta=-2.35)$ QLF in Paper III, the estimated number is $\sim 67$, which can be considered as an upper limit. Matsuoka et al. (2018c) report a new measurement of QLF at $z \sim 6$ with a quasar sample covering the magnitude range of $-22 \leqslant M_{1450} \leqslant-29$. For the first time, it shows a clear break in the $z \sim 6$ QLF. This work suggests a flatter faint end slope $(-1.23)$ and a fainter break magnitude (-24.9). We fix $\alpha$ and $M^{*}$ to be -1.23 and -24.9 and refit our $z \sim 6.7$ QLF in Paper III. We obtain a bright end slope of $\beta=-2.51$ and a normalization of $\Phi^{*}=3.69 \mathrm{Gpc}^{-3} \mathrm{mag}^{-1}$. The predicted number of DES quasars based on this new fit is $\sim 55$.

We have discovered six new quasars among the first 30 candidates that we have observed so far in the DES area. There are a total of 380 candidates. Even though we have not yet modeled the selection completeness and these initial observations tend to focus on relatively bright objects, the rate of our discovery suggests a significant number of additional quasars among the candidates await spectroscopic identifications.

In this paper, we present the initial results of our $z>6.5$ quasar survey in the DES $5000 \mathrm{deg}^{2}$ area. Compared with our survey in the Paper III, we are constructing a deeper optical/IR data set by combining the DES optical photometry and VHS, VIKING, ULAS near-IR data, and unWISE mid-IR data. We carried out forced photometry of VHS, VIKING, ULAS, and unWISE images to improve the survey depth. Although this survey is just starting, the four new quasars at $z>6.8$ together with quasars from Paper III have already significantly increased the number of quasars at $z>6.8$. With new IR data and a future DES data release, we will be able to further expand the survey area and depth. For example, the upcoming deeper version of the unWISE coadds will include the fourth year 
NEOWISE images. The completed survey will allow measurements of the reionization-era QLF measurement to reach one magnitude fainter. The quasars discovered in our survey are ideal targets for high-quality multiwavelength follow-up observations to study the properties of supermassive $\mathrm{BH}$ (SMBH), IGM, and SMBH host coevolution.

We thank Aaron Meisner and Dustin Lang for the discussions about the unWISE data, and Sophie Reed for discussions regarding DES quasar survey. J.Y., X.F., and M.Y. acknowledge support from the US NSF grant AST 15-15115 and NASA ADAP grant NNX17AF28G. X.-B.W. and L.J. thank the support of the National Key R\&D Program of China (2016YFA0400703) and the National Science Foundation of China (11533001, 11721303).

We acknowledge the use of the Magellan Clay and Baade $6.5 \mathrm{~m}$ telescopes. This paper also uses data based on observations obtained at the Gemini Observatory, which is operated by the Association of Universities for Research in Astronomy, Inc., under a cooperative agreement with the NSF on behalf of the Gemini partnership: the National Science Foundation (United States), the National Research Council (Canada), CONICYT (Chile), Ministerio de Ciencia, Tecnología e Innovación Productiva (Argentina), and Ministério da Ciência, Tecnologia e Inovação (Brazil). This publication makes use of data products from the Wide-field Infrared Survey Explorer, which is a joint project of the University of California, Los Angeles, and the Jet Propulsion Laboratory/ California Institute of Technology, and NEOWISE, which is a project of the Jet Propulsion Laboratory/California Institute of Technology. WISE and NEOWISE are funded by the National Aeronautics and Space Administration. We acknowledge the use of the DES, the VHS, VIKING, and ULAS data.

Facilities: Blanco (DECam), WISE, VISTA, UKIRT (WFCam), Magellan Clay (LDSS3), Magellan Baade (FIRE), MMT (Binospec), Gemini South (GMOS).

\section{ORCID iDs}

Jinyi Yang (1) https://orcid.org/0000-0001-5287-4242

Feige Wang (iD https://orcid.org/0000-0002-7633-431X

Xiaohui Fan (1) https://orcid.org/0000-0003-3310-0131

Minghao Yue (1) https://orcid.org/0000-0002-5367-8021

Xue-Bing Wu (i) https://orcid.org/0000-0002-7350-6913

Fuyan Bian (ib) https://orcid.org/0000-0002-1620-0897

Linhua Jiang (iD https://orcid.org/0000-0003-4176-6486

Eduardo Bañados (iD https://orcid.org/0000-0002-2931-7824

\section{References}

Abbott, T. M. C., Abdalla, F. B., Allam, S., et al. 2018, ApJS, 239, 18
Bañados, E., Venemans, B. P., Decarli, R., et al. 2016, ApJS, 227, 11 Bañados, E., Venemans, B. P., Mazzucchelli, C., et al. 2018, Natur, 553, 473 Chambers, K. C., Magnier, E. A., Metcalfe, N., et al. 2016, arXiv:1612.05560 Dey, A., Schlegel, D. J., Lang, D., et al. 2019, AJ, 157, 168

Dye, S., Lawrence, A., Read, M. A., et al. 2018, MNRAS, 473, 5113 Edge, A., Sutherland, W., Kuijken, K., et al. 2013, Msngr, 154, 32

Fabricant, D. G., Fata, R. G., \& Epps, H. W. 1998, Proc. SPIE, 3355, 232

Fan, X., Carilli, C. L., \& Keating, B. 2006, ARA\&A, 44, 415

Fan, X., Narayanan, V. K., Lupton, R. H., et al. 2001, AJ, 122, 2833

Fan, X., Wang, F., Yang, J., et al. 2019, ApJ, 870, 11

Flaugher, B., Diehl, H. T., Honscheid, K., et al. 2015, AJ, 150, 150

Honscheid, K., \& DePoy, D. L. 2008, arXiv:0810.3600

Jiang, L., McGreer, I. D., Fan, X., et al. 2016, ApJ, 833, 222

Kashikawa, N., Ishizaki, Y., Willott, C. J., et al. 2015, ApJ, 798, 28

Lang, D. 2014, AJ, 147, 108

Lang, D., Hogg, D. W., \& Mykytyn, D. 2016a, The Tractor: Probabilistic Astronomical Source Detection and Measurement, Astrophysics Source Code Library, ascl:1604.008

Lang, D., Hogg, D. W., \& Schlegel, D. J. 2016b, AJ, 151, 36

Lawrence, A., Warren, S. J., Almaini, O., et al. 2007, MNRAS, 379, 1599

Mainzer, A., Bauer, J., Cutri, R. M., et al. 2014, ApJ, 792, 30

Mainzer, A., Bauer, J., Grav, T., et al. 2011, ApJ, 731, 53

Matsuoka, Y., Iwasawa, K., Onoue, M., et al. 2018a, ApJS, 237, 5

Matsuoka, Y., Onoue, M., Kashikawa, N., et al. 2016, ApJ, 828, 26

Matsuoka, Y., Onoue, M., Kashikawa, N., et al. 2018b, PASJ, 70, S35

Matsuoka, Y., Strauss, M. A., Kashikawa, N., et al. 2018c, ApJ, 869, 150

Mazzucchelli, C., Bañados, E., Venemans, B. P., et al. 2017, ApJ, 849, 91

McMahon, R. G., Banerji, M., Gonzalez, E., et al. 2013, Msngr, 154, 35

Meisner, A. M., Lang, D., \& Schlegel, D. J. 2017, AJ, 154, 161

Meisner, A. M., Lang, D., \& Schlegel, D. J. 2018, RNAAS, 2, 1

Mortlock, D. J., Warren, S. J., Venemans, B. P., et al. 2011, Natur, 474, 616

Planck Collaboration, Akrami, Y., Arroja, F., et al. 2018, arXiv:1807.06205

Reed, S. L., Banerji, M., Becker, G. D., et al. 2019, arXiv:1901.07456

Reed, S. L., McMahon, R. G., Banerji, M., et al. 2015, MNRAS, 454, 3952

Reed, S. L., McMahon, R. G., Martini, P., et al. 2017, MNRAS, 468, 4702

Robertson, B. E., Ellis, R. S., Furlanetto, S. R., \& Dunlop, J. S. 2015, ApJL, 802, L19

Schlafly, E. F., \& Finkbeiner, D. P. 2011, ApJ, 737, 103

Schlegel, D. J., Finkbeiner, D. P., \& Davis, M. 1998, ApJ, 500, 525

Selsing, J., Fynbo, J. P. U., Christensen, L., \& Krogager, J.-K. 2016, A\&A, $585, \mathrm{~A} 87$

Shen, Y., Strauss, M. A., Oguri, M., et al. 2007, AJ, 133, 2222

Simcoe, R. A., Burgasser, A. J., Bernstein, R. A., et al. 2008, Proc. SPIE, 7014 $70140 \mathrm{U}$

Stevenson, K. B., Bean, J. L., Seifahrt, A., et al. 2016, ApJ, 817, 141

Vanden Berk, D. E., Richards, G. T., Bauer, A., et al. 2001, AJ, 122, 549

Venemans, B. P., Bañados, E., Decarli, R., et al. 2015, ApJL, 801, L11

Venemans, B. P., Findlay, J. R., Sutherland, W. J., et al. 2013, ApJ, 779, 24

Wang, F., Fan, X., Yang, J., et al. 2017, ApJ, 839, 27

Wang, F., Wu, X.-B., Fan, X., et al. 2016, ApJ, 819, 24

Wang, F., Yang, J., Fan, X., et al. 2018a, ApJ, 869, 9

Wang, F., Yang, J., Fan, X., et al. 2018b, arXiv:1810.11926

Willott, C. J., Delorme, P., Reylé, C., et al. 2010, AJ, 139, 906

Wright, E. L., Eisenhardt, P. R. M., Mainzer, A. K., et al. 2010, AJ, 140, 1868

Wu, X.-B., Wang, F., Fan, X., et al. 2015, Natur, 518, 512

Yuan, H., Zhang, H., Zhang, Y., et al. 2013, A\&C, 3, 65 